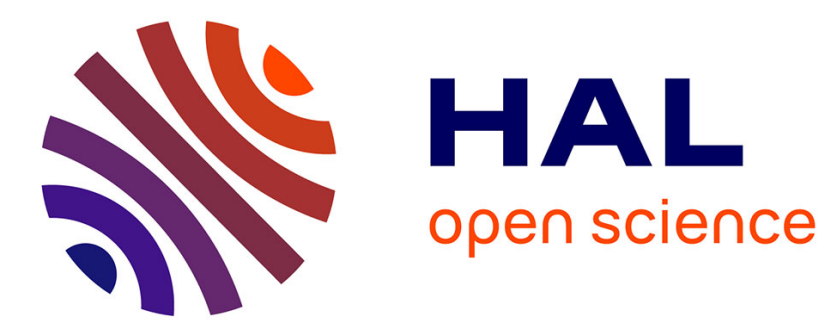

\title{
Analyse in vitro de l'efficacité de la lithotritie laser : quel modèle utiliser ?
}

F. Panthier, Pierre Lapouge, Steeve Doizi, L. Dragos, Laurent Berthe, Olivier Traxer

\section{- To cite this version:}

F. Panthier, Pierre Lapouge, Steeve Doizi, L. Dragos, Laurent Berthe, et al.. Analyse in vitro de l'efficacité de la lithotritie laser : quel modèle utiliser ?. Progrès en Urologie, 2020, 30 (13), pp.709710. 10.1016/j.purol.2020.07.031 . hal-03036563

\section{HAL Id: hal-03036563 https://hal.science/hal-03036563}

Submitted on 2 Dec 2020

HAL is a multi-disciplinary open access archive for the deposit and dissemination of scientific research documents, whether they are published or not. The documents may come from teaching and research institutions in France or abroad, or from public or private research centers.
L'archive ouverte pluridisciplinaire HAL, est destinée au dépôt et à la diffusion de documents scientifiques de niveau recherche, publiés ou non, émanant des établissements d'enseignement et de recherche français ou étrangers, des laboratoires publics ou privés. 
Analyse in vitro de l'efficacité de la

lithotritie laser : quel modèle utiliser ?

F. Panthier ${ }^{1, *}$, P. Lapouge ${ }^{2}$, S. Doizi ${ }^{1}$, L. Dragos ${ }^{1}$, L. Berthe ${ }^{3}$, O. Traxer $^{1}$

$1 G R C n^{\circ} 20$, Groupe de recherche clinique sur la lithiase urinaire, hôpital Tenon, Sorbonne université, Paris, France

2 Laboratoire PIMM, UMR 8006 CNRS, arts et métiers Paris Tech, Paris, France

${ }^{3}$ Laboratoire PIMM, arts et métiers Paris Tech, Paris, France

Auteur correspondant.

*

Adresse e-mail : fredericpanthier@gmail.com (F. Panthier)

Objectifs L'étude in vitro de la lithotritie laser (LL) utilise des calculs humains $(\mathrm{CH})$ ou synthétiques (CS). Devant l'augmentation de la pulvérisation laser (Dusting), peu d'échantillons humains sont disponibles. Les CS sont donc privilégiés, confectionnés par mélange d'eau et de plâtre, avec la problématique de leur réhydratation lors des LL. L'objectif était de comparer les volumes d'ablation (VA) entre $\mathrm{CS}$ homogènes ( $\mathrm{CSHo}$ ) ou hétérogènes $(\mathrm{CSHe})$, et par rapport au poids ablaté (PA).

Méthodes Les CSHo et CSHe durs (COM) ou tendre (UA) étaient confectionnés par mélange de BégostonePlus (Bégo ${ }^{\circledR}$ ) et brassage lent ou rapide avec exsufflation, respectivement. Des fibres laser (Boston Scientific ${ }^{\circledR}$ ) connectées au TFL (IPG Photonics ${ }^{\circledR}$ ) $50 \mathrm{~W}$ $(272 \mu \mathrm{m}$ ou $150 \mu \mathrm{m})$ ou au MH1 Ho :YAG $30 \mathrm{~W}$ (Rocamed $\left.^{\circledR}\right)(272 \mu \mathrm{m})$ ont été utilisées selon trois paramètres laser («fine dusting » [FD : $0,15 \mathrm{~J} / 100 \mathrm{~Hz}]$, «dusting » $[\mathrm{D}: 0,5 \mathrm{~J} / 30 \mathrm{~Hz}]$ et «fragmentation » [Fr : $1 \mathrm{~J} / 15 \mathrm{~Hz}])$. Une émission laser de vingt secondes était réalisée respectant une spirale de rayon $4 \mathrm{~mm}$ en contact avec les CS cubiques de $10 \mathrm{~mm}$, préalablement pesés et immergés. Après séchage, les calculs étaient pesés et scannés (Quantum FX, Perkin Elmer). Les volumes d'ablation étaient mesurés par segmentation (3DSlicer [NIH]) (Fig. 1). La corrélation PA-VA (CPA-VA) était évaluée pour chaque groupe.

Résultats Avec les CSHe, les VA en Dusting étaient quatre et trois fois supérieurs avec le TFL par rapport au Ho :YAG contre COM $(p<0,05)$ et UA $(p<0,05)$. En Fragmentation, les VA étaient deux fois supérieurs avec le TFL par rapport au Ho :YAG contre $\operatorname{COM}(p<0,05)$ et UA $(p<0,05)$ respectivement (Tableau 1). Avec les CSHo cette différence n'était plus significative contre UA en Dusting $(p=0,08)$ et Fragmentation $(p=0,09)$. L'utilisation des CSHo était associée à des différences non significatives entre TFL-150 $\mu \mathrm{m}$ et TFL- $272 \mu \mathrm{m}$, et entre TFL-150 $\mu \mathrm{m}$ et Ho :YAG-272 $\mu \mathrm{m}$. La CPA-VA était supérieure en cas de CSHo par rapport au CSHe $(0,853$ versus $0,428, p<0,0001)$. Cette différence était également retrouvée pour les COM et UA (Tableau 2). Si la CPA-VA était acceptable en cas de COM (CSHo ou 


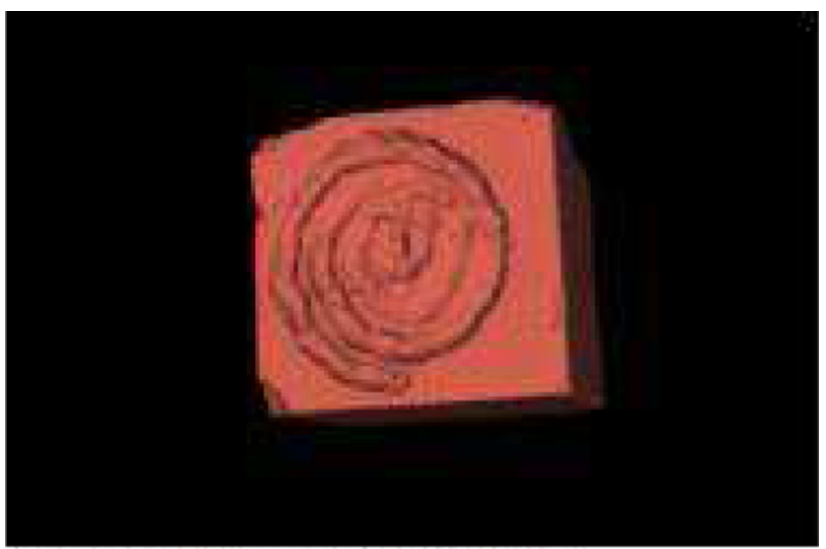

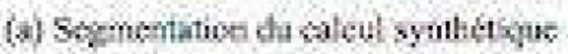

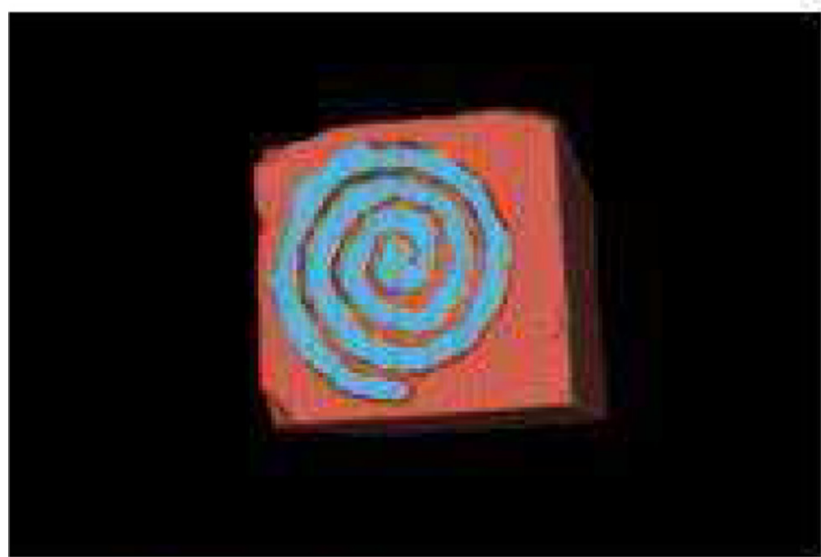

(b) Segmontaíon de l'sir coefers dan le conière

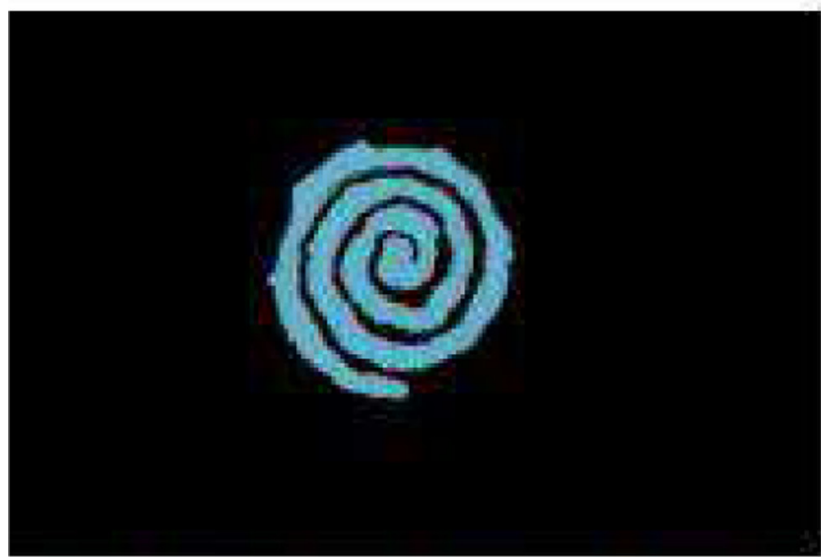

1010 peration kgeque de soustractwe pour ablene to whime distision

Fig. 1 Méthode de segmentation 3D utilisant un logiciel opensource 3DSlicer pour la mesure des volumes d'ablation : (a) segmentation du calcul synthétique (b) segmentation de l'air contenu dans le cratère $(c)$ opération logique de soustraction pour obtenir le volume d'ablation.
Tableau 1 Taux d'ablation.

\begin{tabular}{|c|c|c|c|c|c|}
\hline \multirow{2}{*}{\multicolumn{2}{|c|}{ INTERFACE }} & \multirow{2}{*}{\begin{tabular}{|c}
$\begin{array}{c}\text { PARAMETRES } \\
\text { LASER }\end{array}$ \\
\end{tabular}} & \multicolumn{3}{|c|}{ TAUX D'ABLATION $\left(\mathrm{mm}^{3} / \mathrm{min}\right)$} \\
\hline & & & TFL $150 \mu \mathrm{m}$ & TFL $272 \mu \mathrm{m}$ & p-value \\
\hline \multirow{6}{*}{ CSHo } & \multirow{3}{*}{$\mathrm{COM}$} & Fine Dusting & $41,56 \pm 10,3$ & $51,47 \pm 10,6$ & 0,39 \\
\hline & & Dusting & $50,59 \pm 19$ & $83,54 \pm 13,5$ & 0,11 \\
\hline & & Fragmentation & $65,97 \pm 13,1$ & $77,31 \pm 17,8$ & 0,22 \\
\hline & \multirow{3}{*}{ UA } & Fine Dusting & $44,34 \pm 16,8$ & $51,95 \pm 20,3$ & 0,40 \\
\hline & & Dusting & $42,38 \pm 12,5$ & $47,02+4,08$ & 0,60 \\
\hline & & Fragmentation & $52,48+8,96$ & $55,3 \pm 10,1$ & 0,44 \\
\hline \multirow{6}{*}{ CSHe } & \multirow{3}{*}{$\mathrm{COM}$} & Fine Dusting & $35,68 \pm 0,97$ & $45,93+5,60$ & 0,04 \\
\hline & & Dusting & $38,38 \pm 6,44$ & $61,1 \pm 7,88$ & 0,01 \\
\hline & & Fragmentation & $41,21 \pm 4,04$ & $66,96 \pm 11,39$ & 0,03 \\
\hline & \multirow{3}{*}{ UA } & Fine Dusting & $37,77 \pm 9,24$ & $44,88 \pm 3,41$ & 0,28 \\
\hline & & Dusting & $41,12 \pm 5,61$ & $62,88 \pm 9,25$ & 0,04 \\
\hline & & Fragmentation & $50,16 \pm 11,7$ & $66,57 \pm 10,8$ & 0,01 \\
\hline \multirow{2}{*}{\multicolumn{2}{|c|}{ INTERFACE }} & \multirow{2}{*}{$\begin{array}{l}\text { PARAMETRES } \\
\text { LASER } \\
\end{array}$} & \multicolumn{3}{|c|}{ TAUX D'ABLATION $\left(\mathrm{mm}^{2} / \mathrm{min}\right)$} \\
\hline & & & TFL $150 \mu \mathrm{m}$ & $\mathrm{Ho}_{0} 272 \mu \mathrm{m}$ & p-value \\
\hline \multirow{4}{*}{ CSHo } & \multirow{2}{*}{$\operatorname{COM}$} & Dusting & $50,59+19$ & $22,96 \pm 5,46$ & 0,08 \\
\hline & & Fragmentation & $65,97 \pm 13,1$ & $27,16 \pm 0,75$ & 0,01 \\
\hline & \multirow{2}{*}{ UA } & Dusting & $42,38 \pm 12,5$ & $28,63 \pm 10,2$ & 0,02 \\
\hline & & Fragmentation & $52,48 \pm 8,96$ & $41,16 \pm 8,50$ & 0,18 \\
\hline \multirow{4}{*}{ CSHe } & \multirow{2}{*}{ COM } & Dusting & $38,38 \pm 6,44$ & $16,26 \pm 1,17$ & 0,01 \\
\hline & & Fragmentation & $41,21 \pm 4,04$ & $31,74 \pm 4,60$ & 0,02 \\
\hline & \multirow{2}{*}{ UA } & Dusting & $41,12 \pm 5,61$ & $22,99 \pm 1,23$ & 0,003 \\
\hline & & Fragmentation & $50,16 \pm 11,7$ & $38,6 \pm 5,34$ & 0,12 \\
\hline \multirow{2}{*}{\multicolumn{2}{|c|}{ INTERFACE }} & PARAMETRES & \multicolumn{3}{|c|}{ TAUX D'ABLATION $\left(\mathrm{mm}^{3} / \mathrm{min}\right)$} \\
\hline & & LASER & Ho $272 \mu \mathrm{m}$ & TFL $272 \mu \mathrm{m}$ & p-value \\
\hline \multirow{2}{*}{ CSHo } & \multirow{2}{*}{ COM } & Dusting & $22,96 \pm 5,46$ & $83,54 \pm 13,5$ & 0,001 \\
\hline & & Fragmentation & $27,16 \pm 0,75$ & $77,31 \pm 17,8$ & 0,01 \\
\hline \multirow{2}{*}{$\mathrm{CSHO}$} & \multirow{2}{*}{ UA } & Dusting & $28,63 \pm 10,2$ & $47,02 \pm 4,08$ & 0,08 \\
\hline & & Fragmentation & $41,16 \pm 8,50$ & $55,3 \pm 10,1$ & 0,09 \\
\hline \multirow{2}{*}{ CSHe } & \multirow{2}{*}{$\mathrm{COM}$} & Dusting & $16,26 \pm 1,17$ & $61,1 \pm 7,88$ & 0,002 \\
\hline & & Fragmentation & $31,74 \pm 4,60$ & $66,96 \pm 11,39$ & 0,02 \\
\hline \multirow{2}{*}{$\mathrm{CSHe}$} & \multirow{2}{*}{ UA } & Dusting & $22,99 \pm 1,23$ & $62,88 \pm 9,25$ & 0,003 \\
\hline & & Fragmentation & $38,6 \pm 5,34$ & $66,57 \pm 10,8$ & 0,007 \\
\hline
\end{tabular}

Tableau 2 Coefficient de corrélation entre poids et volume ablaté selon le type de calcul synthétique (CSH) dur (COM) ou tendue (UA), homogène ( $\mathrm{CSHo})$, hétérogène ( $\mathrm{CSHe})$.

\begin{tabular}{|c|c|c|c|cc|cc|c|c|}
\hline COEFFICIENT DE & \multicolumn{3}{|c|}{ UA } & \multicolumn{3}{c|}{ COM } & \multicolumn{3}{c|}{ Tous CSH confondus } \\
\cline { 2 - 10 } $\begin{array}{c}\text { CORRELATION } \\
\text { POIDS-VOLUMM }\end{array}$ & CSHo & CSHe & p-value & CSHo & CSHe & p-value & CSHo & CSHe & p-value \\
\hline
\end{tabular}

CSHe), il n'est pas possible d'utiliser l'approximation du VA par le PA en cas d'UA.

Conclusion L'efficacité in vitro d'une LL est plus précise en utilisant les VA plutôt que les PA. L'approximation du VA par le PA est acceptable et moins coûteuse en cas de CSHo et dur (COM) bien que les calculs urinaires ressemblent plus aux CSHe (COM ou UA). Déclaration de liens d'intérêts Bourse de Recherche AFU 2018. 